\title{
MODELAGEM DA SEDIMENTAÇÃO DE SÓLIDOS ADENSANTES EM SUSPENSÕES AQUOSAS
}

\author{
L. M. M. SILVA ${ }^{1}$, J. M. RIBEIRO Jr ${ }^{2}$, L. A. CALÇADA ${ }^{3}$, C. M. $\operatorname{SCHEID}^{3}$ e \\ L. A. C. MELEIRO ${ }^{3}$
}

${ }^{1}$ Universidade Federal Rural do Rio de Janeiro, Discente do Curso de Engenharia Química

${ }^{2}$ Univ. Federal Rural do Rio de Janeiro, Programa de Pós-graduação em Engenharia Química

${ }^{3}$ Universidade Federal Rural do Rio de Janeiro, Departamento de Engenharia Química

E-mail para contato: meleiro@ufrrj.br

\begin{abstract}
RESUMO - Na indústria petrolífera há uma série de cuidados necessários para manter a segurança e o bom funcionamento dos poços de petróleo. Com relação aos fluidos de perfuração utilizados, a sedimentação das partículas sólidas dos adensantes neles presentes causa flutuações indesejáveis na densidade do fluido, podendo causar problemas, gerar acidentes e até mesmo a perda da linha de perfuração. Nesse sentido, o conhecimento do perfil de sedimentação dos sólidos presentes no fluido de perfuração utilizado tem grande importância para evitar problemas no processo. Entretanto, existe uma dificuldade para se encontrar um modelo de predição da taxa de sedimentação das partículas sólidas para qualquer suspensão, exceto por alguns modelos fenomenológicos de grande complexidade matemática usualmente propostos a literatura. Assim, este trabalho teve como objetivo avaliar uma proposta alternativa de modelagem para a sedimentação de suspensões aquosas de barita utilizando um modelo empírico simplificado, visando aplicá-lo, posteriormente, para a predição da taxa de sedimentação das partículas sólidas em fluidos de perfuração.
\end{abstract}

\section{INTRODUÇÃO}

O processo de sedimentação gravitacional ocorre devido ao deslocamento, pela ação da gravidade, da fase mais densa de uma mistura ou suspensão. Na indústria petrolífera, o processo de sedimentação dos sólidos suspensos em fluidos de perfuração de poços de petróleo pode acabar causando grandes problemas, inclusive acidentes. Isso ocorre devido ao fato da sedimentação gerar flutuações indesejáveis na densidade do fluido, o que pode levar ao descontrole da pressão no fundo do poço. Portanto, é de extrema importância conhecer o perfil e a taxa de sedimentação destas partículas sólidas em suspensão no fluido de perfuração utilizado na abertura do poço e os fatores que podem influenciar na sedimentação do mesmo. No entanto, esta informação tem sido obtida através da utilização de modelos matemáticos bastante complexos e de difícil implementação computacional. Assim, o objetivo deste trabalho foi avaliar o desempenho de um modelo matemático simplificado para predição do perfil de sedimentação de adensantes usados no preparo de fluidos de perfuração. O modelo é baseado na adaptação da teoria de sedimentação (Kynch, 1952) proposta por Renko (1998) e o seu desempenho foi testado na modelagem de dados experimentais de sedimentação de barita em suspensões aquosas realizados por Ribeiro Júnior (2016). Este sistema foi escolhido pelo fato da barita ser um dos materiais mais utilizados para aumentar a densidade dos fluidos 
de perfuração na indústria do petróleo e que o conhecimento de sua curva de sedimentação é tido como um dos maiores desafios nesta indústria.

\section{REVISÃO DE LITERATURA}

A sedimentação é o processo de separação sólido-líquido no qual uma suspensão é deixada em repouso (sedimentação em batelada) ou alimentada continuamente num recipiente (sedimentação contínua). Partículas mais pesadas que o fluido tendem a se depositar no fundo do recipiente devido à ação da gravidade (McCabe et al., 2004).

Coe e Clevenger (1916) demonstraram que a sedimentação em batelada de uma suspensão inicialmente homogênea gera quatro zonas de sedimentação: zona de clarificado, zona de concentração inicial constante, zona de transição com concentrações variáveis e zona de compactação. No entanto, segundo Comings (1940), a sedimentação contínua de suspensões compressíveis varia de acordo com a altura da zona de sedimentação e o uso da metodologia proposta por Coe e Clevenger (1916) não é aconselhada.

Para suprir essa deficiência, Kynch (1952) formulou uma teoria para explicar o processo de sedimentação de suspensões com altas concentrações de sólidos. Sua teoria é derivada da equação da continuidade, não sendo necessário o conhecimento detalhado das forças que atuam sobre as partículas. Para isso foram feitas algumas considerações: o efeito de parede sendo ignorado, as partículas possuindo o mesmo tamanho e forma e a concentração de partículas sendo constante na horizontal. O pressuposto básico dessa teoria é que, em qualquer ponto da suspensão, a velocidade de sedimentação de uma partícula depende somente da concentração local da suspensão. Existe uma interface superior de sedimentação, entre a zona de clarificado e a zona de concentração constante, onde a mudança da densidade da suspensão se propaga como ondas do fundo do recipiente até esta interface com velocidades constantes para cada densidade. A velocidade dos sólidos tende a zero na medida em que a densidade aumenta. O conhecimento da interface de sedimentação, juntamente com a concentração inicial das partículas é suficiente para determinar a variação da velocidade de sedimentação dos sólidos. A teoria de Kynch (1952) é sustentada por uma abordagem gráfica simples para determinar a concentração de sólidos nas camadas e por uma correlação que permite determinar a concentração da suspensão em qualquer ponto da curva de sedimentação.

\section{METODOLOGIA}

\subsection{Ensaios de Sedimentação em Batelada}

Os ensaios de sedimentação de adensantes em batelada foram realizados por Ribeiro Júnior (2016) utilizando três suspensões aquosas de barita com concentrações volumétricas iniciais de 6,7\%,12\% e 20\%. Os ensaios foram feitos em uma proveta graduada de 1 litro, onde as suspensões foram homogeneizadas em um misturador Hamilton Beach e, posteriormente, transferidas imediatamente para a proveta, permitindo a sedimentação livre das partículas. A partir deste momento, a altura da interface entre a zona clarificada e a zona de sedimentação livre foi registrada manualmente a cada intervalo de tempo, até que a variação da altura de sólidos sedimentados no fundo da proveta não apresentasse variação perceptível. 


\subsection{Modelagem Matemática}

A modelagem matemática do processo de sedimentação da barita utilizada neste trabalho foi proposta por Renko (1998). Esta modelagem é fundamentada na abordagem gráfica de Work e Kohler (1940) e na teoria de sedimentação de Kynch (1952), porém considerando o efeito de escala - ou seja, a altura inicial da suspensão - sobre a resolução da superfície de suspensão. Renko (1998) demonstrou que sua abordagem inclui as mesmas informações que a tradicional teoria de sedimentação de Kynch (1952), porém com a capacidade de determinar as camadas de sólidos em suspensão, além de eliminar a desvantagem da abordagem de Kynch que demanda um procedimento gráfico lento e enfadonho para a determinação da mesma informação.

De acordo com a hipótese proposta por Renko (1998), a concentração média entre as curvas de sedimentação em um instante de tempo $t$ pode ser calculada a partir da relação:

$$
\left(h_{0}-H_{0}\right) X_{0}=(h(t)-H(t)) X(t, h)
$$

onde $X_{0}$ é a concentração inicial de sólidos; $X(t)$ é a concentração de sólidos no tempo $t ; h(t)$ e $H(t)$ são as alturas das curvas de sedimentação no tempo $t ; X(t, h)$ e $X(t, H)$ são as concentrações médias entre $h(t)$ e $H(t)$ e $H(t)$ e o eixo vertical no tempo $t$, respectivamente, e $h_{0}$ e $H_{0}$ são as alturas iniciais da curva de sedimentação.

A concentração média entre a curva de sedimentação inferior e o eixo horizontal pode ser calculada com a equação:

$$
H_{0} X_{0}=H(t) X(t, H)
$$

Se no tempo $t$ a diferença entre as alturas iniciais de duas curvas for pequena, a expressão proposta por Renko (1998) se reduz a:

$$
X(t, h)=\frac{X_{0}}{\frac{\partial h\left(t, h_{0}\right)}{\partial h_{0}}}
$$

Em um determinado instante de tempo, o perfil de concentração depende somente da concentração inicial de sólidos $\left(X_{0}\right)$, que é uma constante, e da derivada parcial de uma função de $h\left(t, h_{0}\right)$ em relação a $h_{0}$. Assim, a modelagem do perfil de concentração acaba sendo a modelagem da curva de sedimentação, onde o efeito da altura inicial na curva é levado em consideração de acordo com a abordagem de Work e Kohler (1940).

O modelo para a curva de sedimentação proposta por Renko (1998) é dado por:

$$
h\left(t, h_{0}\right)=\frac{C X_{0} h_{0}}{\alpha}+\left(h_{0}-\frac{C X_{0} h_{0}}{\alpha}\right) e^{-t \alpha /\left(X_{0} h_{0}\right)}
$$


onde $h\left(t, h_{0}\right)(\mathrm{m})$ é a altura da interface de clarificação durante a sedimentação no tempo $t(s)$, $h_{0}(\mathrm{~m})$ é altura inicial da curva de sedimentação, $\alpha\left(\mathrm{kg} \mathrm{m}^{-2} \mathrm{~s}^{-1}\right)$ e $C\left(\mathrm{~m} \mathrm{~s}^{-1}\right)$ são os parâmetros que descrevem a sedimentação dos sólidos e $X_{0}$ é a concentração inicial de sólido $\left(\mathrm{kg} \mathrm{m}^{-3}\right)$.

A derivada parcial da equação acima em relação a $h_{0}$ fornece:

$$
\frac{\partial h\left(t, h_{0}\right)}{\partial h_{0}}=\frac{C X_{0}}{\alpha}+\left(1-\frac{C X_{0}}{\alpha}\right)\left(1+\frac{t \alpha}{X h_{0}}\right) e^{-t \alpha /\left(X_{0} h_{0}\right)}
$$

Assim, o modelo para o perfil de concentração de sólidos suspensos é dado por:

$$
X(t, h)=\frac{X_{0}}{\frac{C X_{0}}{\alpha}+\left(1-\frac{C X_{0}}{\alpha}\right)\left(1+\frac{t \alpha}{X_{0} h_{0}}\right) e^{-t \alpha /\left(X_{0} h_{0}\right)}}
$$

O comportamento dos sólidos durante a sedimentação já havia sido estudado anteriormente por Kynch (1952), mas sua teoria apresenta diversas limitações devido às considerações iniciais (como o tamanho e forma das partículas), não podendo, portanto, ser usada para qualquer tipo de suspensão, por isso a necessidade do desenvolvimento de outros métodos. Assim, Renko (1998), baseado nos estudos de Kynch (1952) e Work e Kohler (1940), propôs uma nova abordagem focada na distribuição de sólidos durante a sedimentação em repouso.

A dificuldade de se encontrar um modelo simplificado que possa ser usado na predição da sedimentação de qualquer fluido de perfuração se dá devido às diferenças morfológicas e reológicas entre eles, além de outros fatores físico-químicos e relacionados à geometria do poço. Tal dificuldade só pode ser suplantada através do aumento da complexidade do modelo matemático, o que, por sua vez, demanda métodos numéricos mais sofisticados e mais complexos de solução.

\section{RESULTADOS E DISCUSSÃO}

A modelagem do processo de sedimentação foi realizada utilizando a Equação 4 e a Equação 6. Os parâmetros do modelo foram determinados com auxílio de um conjunto de dados experimentais de sedimentação em batelada obtido por Ribeiro Júnior (2016), que usou suspensões aquosas de barita com concentrações volumétricas iniciais de 6,7\%, $12 \%$ e $20 \%$. Os valores dos parâmetros obtidos para este sistema foram $\alpha=4,5379 \times 10^{-5} \mathrm{~kg} \mathrm{~m}^{-2} \mathrm{~s}^{-1} \mathrm{e}$ $C=1,2048 \times 10^{-4} \mathrm{~m} \mathrm{~s}^{-1}$.

Nas Figuras 1 a 3, apresentadas a seguir, os resultados da modelagem do processo são representados na forma gráfica e ilustram a variação da altura da interface de clarificação em função do tempo e os perfis de concentração em função do tempo e da altura da proveta. 


\section{C*BEEQ \\ XII Congresso Brasileiro de Engenharia Química \\ em Iniciação Científica \\ UFSCar - São Carlos - SP \\ QUIMICA EM INICIAÇÃO CIENTÍFICA \\ 16 a 19 de Julho de 2017}

Figura 1 - Resultado da modelagem proposta para suspensão de barita de 6,7\%.
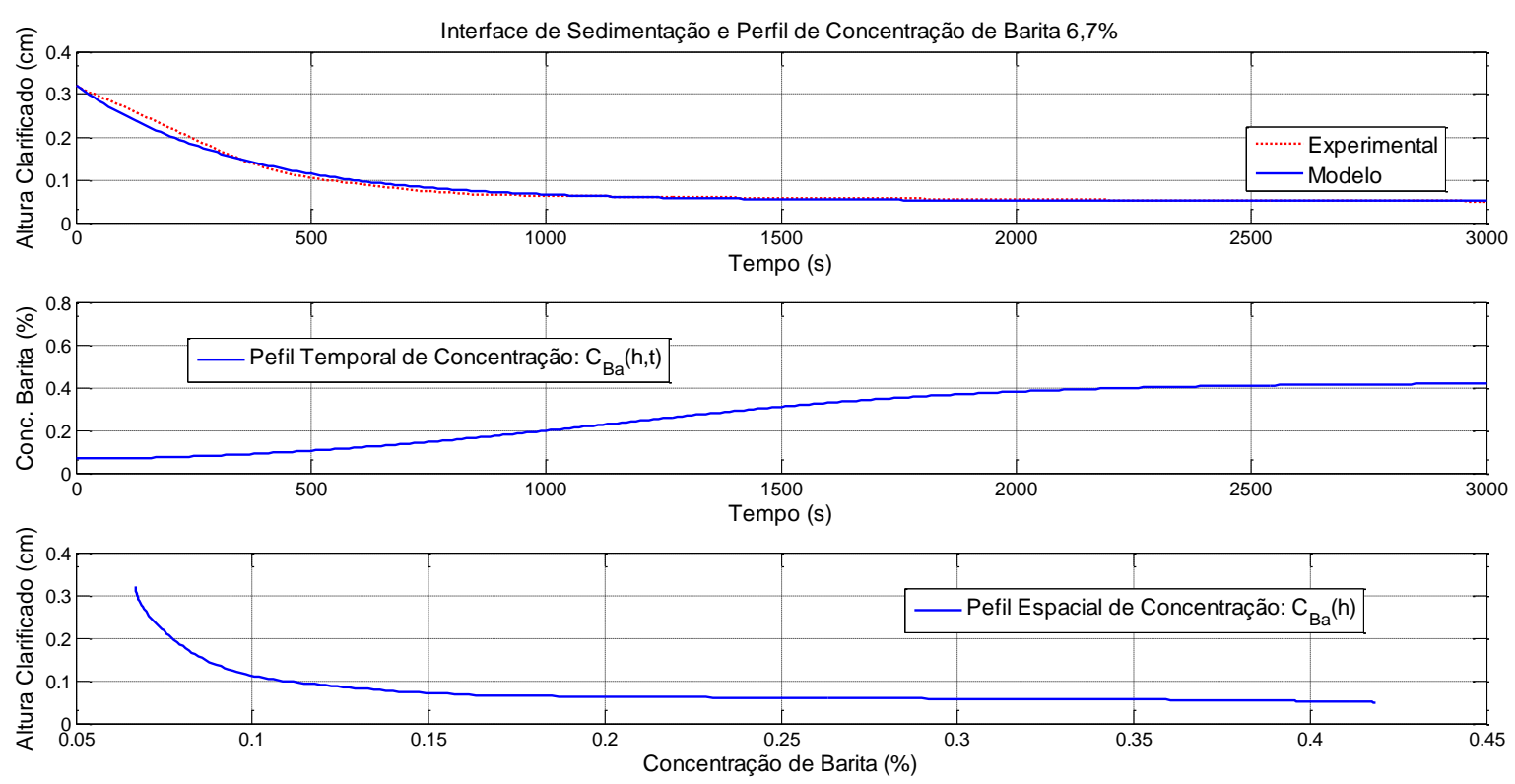

Figura 2 - Resultado da modelagem proposta para suspensão de barita de $12 \%$.
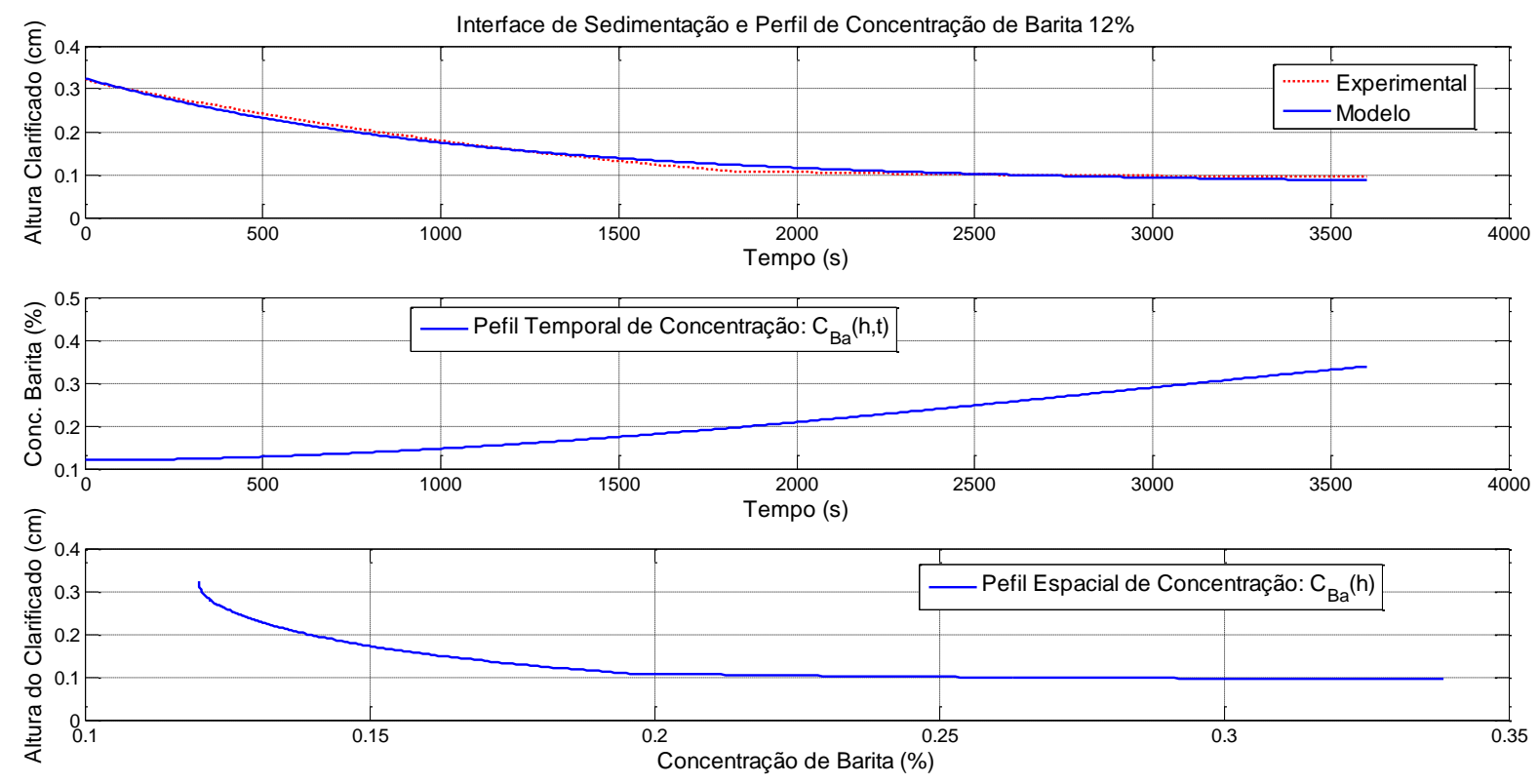

Através dos resultados apresentados nessas Figuras é possível verificar que as predições do modelo para a queda da interface de sedimentação se ajustaram bem aos valores experimentais, visto que os erros de modelagem foram menores do que $10 \%$ para todas as concentrações utilizadas. Além disso, os resultados da simulação apresentaram comportamento qualitativo coerente com os perfis temporais obtidos em laboratório, inclusive na região entre a zona de clarificado e a zona de compactação. Dessa forma, é possível verificar que os resultados obtidos com a modelagem proposta neste trabalho foram bastante satisfatórios e conseguiram prever bem o fenômeno da sedimentação de barita para as três concentrações iniciais de sólido utilizadas nas sedimentações. 
Figura 3 - Resultado da modelagem proposta para suspensão de barita de $20 \%$.
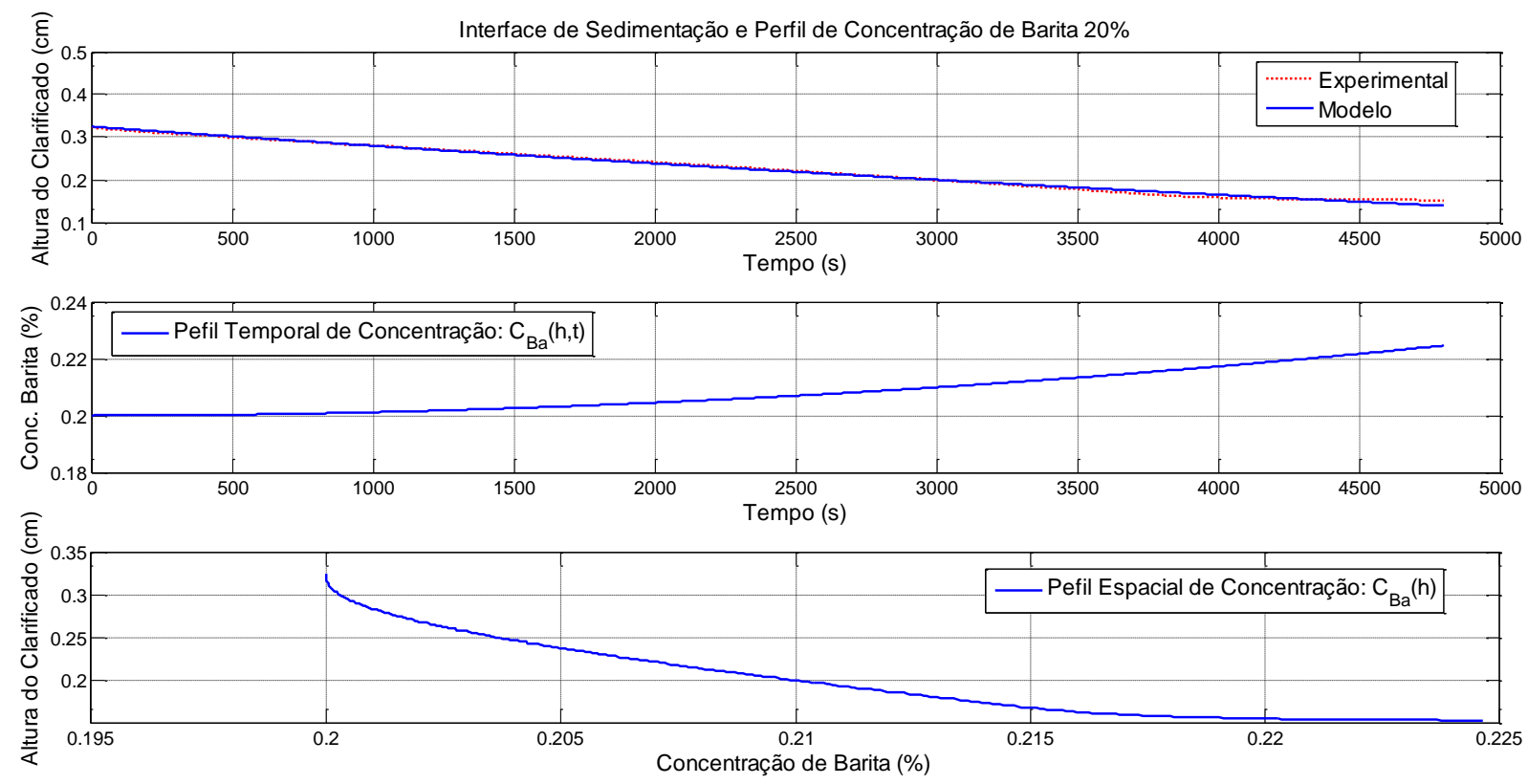

\section{CONCLUSÃO}

Apesar da simplicidade do modelo utilizado neste trabalho para modelar os experimentos realizados em laboratório - nesse caso, a curva de sedimentação da barita -a modelagem matemática proposta por Renko (1998) apresentou um desempenho satisfatório e os parâmetros foram obtidos com relativa facilidade, outro fato que não se verifica quando se utiliza modelos matemáticos mais complexos.

\section{REFERENCIAS}

COE, H. S.; CLEVENGER, G. H. Methods for determining the capacities of slime thickening tanks. AIME Transactions, v. 55, p. 356-384, 1916.

COMINGS, E. W. Thickening calcium carbonate slurries. Journal of Industrial and Engineering Chemistry, v. 32, n. 5, p. 663-668, 1940.

MCCABE, W.; SMITH, J.; HARRIOTT, P. Unit Operations of Chemical Engineering (7th edition), McGraw-Hill, 2004.

KYNCH, G. J. A theory of sedimentation. Trans. Faraday Soc. 48 166-176. 1952

RENKO, E. K. Modelling hindered batch settling Part II: A model for computing solids profile of calcium carbonate. Water SA,Vol. 24, No. 4, 1998.

RIBEIRO JR, J. M. Modelagem e simulação da sedimentação de sólidos adensantes em fluidos de perfuração. 2016. 83p. Dissertação (mestrado) - Universidade Federal Rural do Rio de Janeiro, Curso de Pós-Graduação em Engenharia Química, 2016.

WORK, L.T. and KOHLER, A. S. Sedimentation of suspensions. Ind. and Eng. Chem. 32 1329-1334, 1940. 